\title{
A PSYCHOMETRIC STUDY OF ADOLESCENT RISK PERCEPTION
}

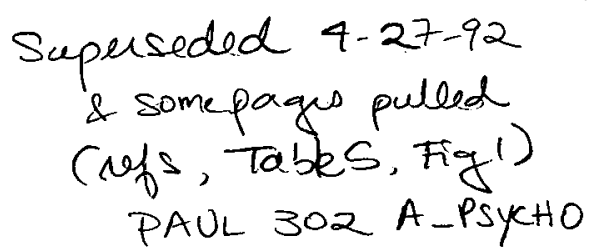

\author{
Alida C. Benthin \\ Department of Human Development and Family Sciences \\ Oregon State University \\ Corvallis, Oregon
}

Paul Slovic

Decision Research

Eugene, Oregon

and

Herbert Severson

Oregon Research Institute

Eugene, Oregon

Address Correspondence To:

Alida C. Benthin

Department of Human Development \& Family Sciences

Milam Hall 322

Oregon State University

Corvallis, OR 97331

Phone: (503) 737-2826 
Psychometric Study of Adolescent Risk Perception

Benthin, Slovic, Severson / page 3

Adolescence is a period of high risk in a person's life. It is the only age group for which mortality has risen since 1960 , with traffic accidents constituting the leading cause of death among people between 15 and 24 years of age (National Center for Health Statistics, 1982). For example, people aged 16-24 accounted for 58\% of all driver fatalities in 1982, even though they represented only about $21 \%$ of the licensed drivers.

Risky driving is only one of many high-risk behaviors that take place during adolescence. Reporting on high-school seniors; Johnston, O'Malley, and Bachman (1989) found that $92 \%$ had tried alcohol, $64 \%$ were current users; and $35 \%$ had five or more drinks in a row at least once during the previous two weeks. Surveys have shown that more than $50 \%$ of all young drivers drink and drive. Phelps (1987) found that, compared with a sober young driver, drinking 1-2 drinks increases the risk of getting into an accident by a factor of 2.3 and six or more drinks raises the relative risk by a factor of 100 . Problem drinking has also been linked to the use of other drugs such as marijuana (Donovan \& Jessor, 1978). Marijuana has been found to be the most widely used illicit drug among adolescents, with $50.9 \%$ of high-school seniors having used the drug and one in 25 seniors smoking it daily (Johnston, O’Malley, \& Bachman, 1987).

In addition to alcohol and illicit drug consumption, the use of tobacco among adolescents remains a serious problem. The percentage of teen-aged youth who smoke at least one cigarette a day is $21.5 \%$, which approaches the level of adult smoking (Severson, 1984). According to a 1986 national survey, three million young people under the age of 21 had used some form of smokeless tobacco (chew or snuff) in the year preceding the survey (Cullen, 1986). 
Psychometric Study of Adolescent Risk Perception

Benthin, Slovic, Severson / page 4

Yet another risk factor during adolescence is teenage pregnancy. According to the National Center for Health Statistics, 36.5 out of every 1000 women aged 15-17 gave birth in 1989, the highest rate since the early 1970's. Increasing sexual activity among teenagers also increases the risk of sexually transmitted diseases. A report in the November 28, 1985 Weekly Surveillance Report (Centers for Disease Control, 1988), stated that 20-29-year-olds make up $21 \%$ of all AIDS cases. Some of these cases may have been infected as adolescents. Although teenagers are at increased risk of becoming infected with sexually transmitted diseases such as AIDS, studies have shown that they do not believe that they are likely to become infected. Results of a 1986 random-sample survey of 860 adolescents aged $16-19$ indicate that only $15 \%$ of all sexually active adolescents in the sample changed their sexual behavior because of concern about contracting AIDS (Strunin \& Hingson, 1987).

The statistics cited above demonstrate that significant numbers of adolescents engage in behaviors that endanger their health and well-being. The risky behaviors noted here are illustrative rather than exhaustive, with the emphasis placed on behaviors that have caused considerable societal concern.

The phenomenon of teenage risk taking has stimulated the development of a socialpsychological framework termed "Problem-Behavior Theory," which addresses some of the most important components of adolescent risk taking (Jessor 1984, 1987; Jessor \& Jessor, 1977). Problem-Behavior Theory focuses on personality factors and environmental forces as major determinants of adolescent risk taking. Each of these components can be broken down into specific sub-components which may either inhibit or encourage risk taking among young people. 
Findings from studies guided by Problem Behavior Theory have drawn attention to a syndrome of adolescent problem behavior. Specifically, these studies have shown that risky behaviors tend to be interrelated rather than being a collection of independent activities. The present article proposes to extend Problem Behavior Theory to include adolescent risk perception. Specifically, it is proposed that adolescent risk taking is, in part, influenced by young people's perceptions and attitudes concerning the riskiness involved in a certain activity.

The present study employs a psychometric paradigm that has been widely used to investigate perceived risk. This approach uses psychophysical scaling and multivariate analysis to produce "cognitive maps" of risk perceptions (Slovic, 1987). Within this paradigm, people make quantitative judgments about the riskiness of various hazardous activities and technologies. These judgments are then related to judgments about a number of risk characteristics that have been hypothesized to influence risk perceptions.

A variety of studies of risk perception have been conducted within the psychometric paradigm (Johnson \& Tversky, 1984; Slovic, Fischhoff, \& Lichtenstein, 1979, 1986; among others). Although the results of these studies have sometimes differed, there has been enough consistency across studies to distill some generalizations about perceived risk. Central among these is that perceived risk is quantifiable and predictable (Slovic, 1987).

The earliest psychometric surveys by Fischhoff, Slovic, Lichtenstein, Read, and Combs (1978) and Slovic, Fischhoff, and Lichtenstein (1979) asked people to rate 30 different activities and technologies with regard to their perceived riskiness. In addition, survey respondents were asked to judge each of the 30 hazards with regard to nine characteristics which were though to 
be important for the way people perceive risk. These characteristics were (a) whether the risk is assumed voluntarily or involuntarily; (b) whether the risk of death from the hazard is immediate or delayed; (c) whether the risks are known to those exposed; (d) whether the risks are known to science; (e) whether risks from the hazard can be controlled by the individual; (f) whether the risk is new (unfamiliar) or old (familiar); (g) whether an accident is likely to kill one person at a time (chronic) or large numbers of people at once (catastrophic); (h) whether the thought of the risk evokes a feeling of dread and (i) whether an accident caused by an activity is likely to result in fatalities. Respondents were asked to rate each of the 30 activities and technologies on all nine characteristics using a seven-point bipolar scale. The nine characteristics were found to predict whether a particular risk was acceptable (Slovic, Fischhoff, \& Lichtenstein, 1979). Another of the findings in these studies was that the concept "risk" has different meanings for experts than for laypersons (Slovic, 1987).

The present study is the first attempt to employ the psychometric paradigm to assess how young people think about risk. The results will indicate whether the quantitative assessment methodology that has successfully predicted risk perceptions and social response to risk in college students and adults can be successfully applied with adolescents. In addition to describing perceptions quantitatively, the present study will examine the relationships between perceptions and self-reported participation in high-risk activities. 
Psychometric Study of Adolescent Risk Perception

Benthin, Slovic, Severson / page 7

\section{METHOD}

Sample. Participants in this study were 41 student volunteers from two high schools in Eugene, Oregon. They were recruited through announcements in class and by word of mouth. Of those participants who indicated gender, 25 were females and 15 were males. Their mean age was 15.5 years (range 14 to 18 ). Each person was paid $\$ 10$ for participation.

Procedures. Parent permission slips were sent home with the students three weeks before the data were collected. All participants returned the permission slips. Subjects responded to a self-report questionnaire after school at a local research institute. On average it took 30 minutes to complete the questionnaire. Participants were assured of complete confidentiality in the treatment of the data and were asked not to write their names on the questionnaires.

Design. Quantitative judgments were made for 30 risk behaviors. As indicated in Table 1, these behaviors included 11 items considered to represent problem behaviors (e.g., smoking marijuana, using cocaine, etc.) and 19 items representative of what might be considered socially approved risk-taking behaviors (e.g., swimming, riding a bicycle, etc.). The goal in selecting these items was to provide a comprehensive context in which to compare and evaluate risk perceptions. Items were selected according to several criteria including prevalence, popularity and presumed importance from a risk standpoint. Some of the items, however, pose much greater risks than others (e.g., using crack vs. ingesting caffeine). The risks from engaging in sexual activity were rated separately for risk of pregnancy and risk of disease.

Insert Table 1 about here 
Each of the 30 items was rated by each respondent on the 14 risk characteristics shown in Table 2. Some of these risk characteristics were selected because of their importance in previous studies of risk perception (e.g., knowledge, warning sign, etc.), whereas others were selected because of their potential relevance to adolescent behavior (e.g., peer influence, admiration, etc.). All 30 risk behaviors were rated on one risk characteristic before the next characteristic was considered. Each rating used a seven-point scale. Respondents were also asked to indicate how many times they themselves had engaged in each of the 30 activities during the previous six months by checking one of four frequency categories (none, once or twice, 2-5 times, $>5$ times).

Insert Table 2 about here

\section{RESULTS}

\section{Mean Ratings}

Table 3 presents the means and standard deviations for each rating scale, along with the extreme high- and low-rated activities for each scale. The avoidability scale had the highest overall mean (5.73) with a range from 4.24 to 6.78 , indicating that our respondents thought that all 30 activities are moderately to highly avoidable for young people. A rather low mean for perceived peer influence $(2.44)$ reflected the perception that peer influence plays only a minor role in young people's motives to engage in these risk-taking activities. The lowest specific means for peer influence occurred for the use of hard drugs such as methamphetamines, crack, 
and cocaine and the highest mean (only 4.20 ) was associated with driving a car. The means on the various risk and benefit scales (fear, personal risk, risk to peers, benefits vs. risks) varied greatly across the 30 activities. Hard drugs, such as crack and cocaine, were perceived to be most frightening and least beneficial relative to their risks; bicycle riding was viewed most favorably in terms of low perceived risk and a high benefit vs. risk rating. The overall means for personal risk (3.95) and risk to peers (3.94) were quite similar and the two sets of means correlated .99 across the 30 activities. The overall mean for perceived knowledge of the risks (4.0) and the small standard deviation (0.81) across the 30 items indicates that the risks associated with most of the activities were perceived as moderately well known among adolescents. Of particular interest in this context was that "having sex" received the highest mean rating (5.22) among all 30 activities, suggesting that the young people in our sample thought that the risks of having sex are particularly well known among young people. In addition, our respondents thought that the risks of sex are about as controllable as risks from sports and swimming and more controllable than the risks from driving a car.

Insert Table 3 about here

Mean evaluations for two types of risk associated with sexual activity (risk of pregnancy and risk of disease) showed that risks from pregnancy (5.56) were perceived to be better known than risks from disease $(4.88 ; \mathrm{p}<.01)$ and that the risk of disease $(5.73)$ was more feared than the risk of pregnancy $(4.95 ; \mathrm{p}<.01)$. Our respondents perceived their peers to be at more risk 
from pregnancy than themselves, whereas the mean risk from disease was about the same for self and others. Furthermore, the risk from disease was perceived as slightly less controllable than the risk from pregnancy. The benefit/risk balance was negative for both risks, particularly for risk of disease.

\section{Intercorrelations Among Activities}

Based upon their confidential self reports, each respondent was categorized as someone who did or did not participate in each of the activities. These dichotomous categorizations were then intercorrelated across the 41 individuals. Table 4 presents these intercorrelations for 11 high-risk activities. ${ }^{1}$ A positive correlation between a pair of activities indicates that people who engage in one also tend to engage in the other. The finding that 50 out of 55 correlations among the 11 high-risk activities were positive, strongly suggests a syndrome of problem behavior. For example, young people who were found to drink alcohol were also relatively more likely to smoke cigarettes $(r=.32)$ and to use marijuana $(r=.57)$. Unprotected sexual behavior was associated with use of snuff and drug taking. A high correlation was observed between the use of "uppers" and the use of cocaine. Use of crack is the only activity that showed negative correlations with other behaviors, and these correlations were quite small.

\section{Insert Table 4 about here}

${ }^{1}$ These correlations were computed by coding the four frequency categories on a 1-4 scale. Note that there were two distinct questions pertaining to sexual activity. One question asked how frequently the respondent had engaged in sex during the previous six months. The second asked about frequency of unprotected sex, defined as sex without a condom. 
Psychometric Study of Adolescent Risk Perception

Benthin, Slovic, Severson / page 11

\section{Comparisons Between Participants and Non-participants}

There were a number of strong differences in the perceptions of those who participate in an activity and those who do not. Generally participants reported greater knowledge of the risks from the activities they perform, less fear of those risks, less personal risk, less risk to peers, greater benefits relative to risks, less seriousness of effects, more personal control over the risks, greater peer pressure, less desire for regulation, less ability to avoid doing the activity and higher perceived participation rate by peers.

Despite the small sample sizes in the participant and non-participant groups, many of the differences for high-risk behaviors such as excessive consumption of alcohol, smoking cigarettes, using marijuana, and engaging in sexual activity, were statistically significant, as shown in Table 5. Similar patterns of differences were also found for socially approved risk taking such as climbing, skateboarding, and skiing.

\section{Insert Table 5 about here}

\section{Factor Analysis}

The intercorrelations among all 14 of the risk characteristics (personal risk, risk to peers, etc.) were subjected to a principle component factor analysis. The analysis produced two uncorrelated factors, with the first factor accounting for $68 \%$ of the variance among the risk characteristics and the second factor accounting for $15 \%$ of the variance. After varimax rotations the pattern of factor loadings indicated that Factor 1 was primarily determined by 
perceived risk, fear, and perceived seriousness of consequences, whereas Factor 2 was determined by admiration and knowledge and, to a lesser extent, peer influence. We have chosen to name Factor 1 "Risk" and Factor 2 "Admiration." It is interesting to see that knowledge loads highly on the same factor as admiration. This reflects a correlation of .49 between the means for these two characteristics across the 30 activities. The most admired activities (driving, contact sports, skiing, and sex) were also judged to have risks that were relatively well known, and many of the least admired activities (eating disorders, use of pain relievers) were judged to pose risks that were not well known.

Factor scores were computed for each of the 30 activities by weighting the ratings on each risk characteristic proportional to the importance of the characteristic for the factor and summing over all characteristics. This weighted sum gives a particular activity a score that is an amalgamation of its ratings on the variables that define the factor. The factor scores for each activity are plotted in Figure 1. As one moves from left to right in the space, the activities are judged to be more risky to self and others. As one goes from the bottom to the top of the space, the activities are judged to be more admired and better known. It is interesting to note that many of the most dangerous activities (upper-right quadrant; both actual danger and perceived risk) are as highly admired as the more socially approved forms of risk taking (upper-left quadrant).

Insert Figure 1 about here 
Psychometric Study of Adolescent Risk Perception

Benthin, Slovic, Severson / page 13

\section{DISCUSSION}

One message in these data is that a methodology which had previously been used to investigate perceived risk only in college students or adult populations can also be used with adolescents. Although the present results need to be generalized with caution because of the small, self-selected nature of the sample, the finding that the results were orderly and meaningful leads to the general conclusion that we have developed a useful psychometric instrument to investigate how adolescents view risk.

One important finding from this study is that actual participation in risky activities is related to very distinct cognitive and social factors. From a cognitive perspective, people who engage in the activity report greater knowledge of risks, less fear of risks, less risk to self and others, less seriousness of effects, more personal control over risk, less ability to avoid the activity and higher participation in the activity by others. The finding that those who participate in a given activity also perceive a generally higher participation rate is consistent with the availability heuristic that has been found to determine judgments of frequency and probability (Tversky \& Kahneman, 1973). By this heuristic, the estimation of the frequency of a behavior is mediated by the ease with which instances or associations of the behavior can be brought to mind through imagination or recall. Obviously, participants in an activity would have greater ability to recall or imagine the activity being performed.

From a social perspective, participants report greater peer influence, less desire for regulation of the activity by authorities and greater benefits relative to risks. The importance of peer influence has long been acknowledged as a major variable in adolescent risk taking. For 
example, Jessor and Jessor (1975) found that young people who engage in high-risk behavior perceived greater support for their risk taking behavior from friends and also reported having more friends who engage in such behavior. The finding that risk taking may actually provide benefits for the young person requires specific attention. Although it has been suggested that engaging in risky behaviors may serve a variety of functions from leading to social maturity to establishing identity (Baumrind, 1987; Irwin \& Millstein, 1986; Jessor, 1984), it is still unclear why certain behaviors yield relatively high benefits for some teenagers whereas they remain less attractive for others. Jessor (1984) points out that some young people may use excessive risktaking behavior as a way to achieve goals that may seem unattainable otherwise. For example, risk taking may be seen by the adolescent as an effective way to gain independence from parental control.

It is also notable that results from this study point to a problem behavior syndrome. In other words; young people who engage in one form of risk taking behavior (e.g., excessive use of alsochol). are relatively more likely to engage in another (e.g., smoking cigarettes). These results are in line with previous research, suggesting that problem behaviors during adolescence tend to be interrelated rather than being a collection of independent activities (Biglan, Wendler, Wirt, Ary, Noell, Ochs, French, \& Hood, 1990; Donovan \& Jessor, 1985; Jessor, 1984).

The finding that those who engage in risky activities tend to perceive the risks as well known, raises questions about the potential effectiveness of information and educational programs designed to discourage young people from engaging in risk-taking behaviors. Do adolescents actually have adequate risk information? Do they simply have a general idea that 
the activity is dangerous, or are they actually knowledgeable about specific risks? Do they understand that certain risks may apply to them personally and do they know how they can protect themselves from potential harm if they engage in risky behavior? Finally, will they ignore information or education programs on the grounds that they think they already know the risks?

The finding that participants in high risk activities thought that they could control the risks but at the same time believed they were unable to avoid the activity is also intriguing. Why do some young people believe they cannot control their engagement in an activity, but, once involved, believe they can control its risks? The former belief may stem from sensitivity to peer pressures which may be hard to resist. The latter belief may point to a "personal fable" of uniqueness and immortality, which has been said to be a typical dimension of adolescent thinking (Seltzer, 1982). So far, the notion of a personal fable has not been empirically verified and future research needs to determine whether such a fable actually exists, whether it is part of a normal developmental course in adolescence, and whether it is more pronounced in some young people than in others.

Many other future directions are indicated for this type of research. It would be interesting, for example, to investigate whether young people differ in their perceptions of risk from adults. Although such a difference is generally presumed in the adult population, it has yet to be empirically verified. It may well be that young people are similar to older people in their perceptions of risk and that perceived differences derive from the fact that certain activities 
(e.g., drinking) are not socially approved for younger populations whereas they are encouraged among adults.

Research is particularly needed to determine why young people who participate in highrisk activities differ from their nonparticipating peers in their perceptions of the risks and benefits of these activities. Longitudinal studies are needed to assess the development of perceptions and to determine if the perceptions associated with risk-taking behaviors occur prior to engagement in the behaviors (and thus can be used to predict the onset of these behaviors). An alternative hypothesis, suggested by dissonance theory (Festinger, 1957) and also testable longitudinally, is that the behavior drives the perceptions, rather than vice-versa. Adolescents who participate in high-risk activities may be motivated to reduce dissonance or rationalize their actions by making their beliefs consistent with their behaviors.

Research might also examine the relationship between adolescent risk taking and the development of formal operational thinking characterized by the ability to think abstractly (e.g., about risk) and to apply hypothetical and deductive reasoning (Inhelder \& Piaget, 1958). Damody (1991), for example, found that adolescents deficient in formal reasoning ability were less able to plan ahead or delay gratification.

Another hypothesis in need of testing is that repeated exposure to a stimulus is a sufficient condition to enhance an observer's favorable attitude toward that stimulus (Zajonc, 1968). Thus adolescents who are frequently exposed to risky activities (e.g., via the peer group) may come to perceive these activities as less threatening than those who are not as frequently 


\section{Psychometric Study of Adolescent Risk Perception}

Benthin, Slovic, Severson / page 17

exposed to the activity. This may be particularly true for activities such as smoking, whose harmful effects are not immediately apparent.

Drawing on these ideas, future research needs to examine adolescent risk perceptions in light of the social context (e.g., family, peer group, school, etc.) in which they develop. A contextual understanding of adolescent risk perceptions may be required to understand the social reality of the adolescent to the degree necessary for the development of effective prevention and intervention strategies. 
Psychometric Study of Adolescent Risk Perception

Benthin, Slovic, Severson / page 22

\section{ACKNOWLEDGEMENT}

This research was supported by a grant from the Alcoholic Beverage Medical Research Foundation, Herb Severson and Paul Slovic, co-investigators. We are grateful to Mark Layman and Maureen Barkley for assistance with data analysis and to Sarah Hampson for helpful comments on the manuscript. 
Table 1. Items Used in the Present Study

1. Problem Behaviors

Drinking Alcoholic Beverages

Smoking Cigarettes

Smoking Marijuana

Drinking Five or More Alcoholic Beverages

Using Crack

Taking Methamphetamines (Uppers)

Using Cocaine

Riding in a Car with an Intoxicated Driver

Using Smokeless Tobacco

Binge Eating/Vomiting

Crash, or Compulsive Dieting

2. Other Behaviors

Climbing Mountains or Rocks

Riding Motorcycles

Driving an All-Terrain Vehicle

Swimming

Riding a Bicycle

Playing Contact Sports

Taking Prescription Drugs

Riding Skateboards

Downhill Skiing

Driving a Car

Sunbathing

Using Caffeine

Using Artificial Sweeteners

Using Fireworks

Taking Pain Relievers

Taking Diet Pills

Listening to Loud Music

Having Sex

Hitchhiking 
Table 2. Characteristics on which the 30 Items were Rated

\section{OLD OR NEW RISK}

Is this a new type of risk for people your age, or an old one that has been around for a long time? $(1=$ very new; $7=$ very old $)$

\section{KNOWLEDGE OF ADOLESCENTS}

To what extent are the risks associated with this activity known to people your age? ( 1 = risks not well known; 7 = risks very well known)

\section{FEAR}

To what extent are the potential risks (dangers) associated with this activity frightening for people your age? ( $1=$ not at all frightening; $7=$ very frightening)

\section{PERSONAL RISK}

If you did this activity, to what extent do you believe that you would be personally at risk of getting hurt or getting sick? ( $1=$ very much at risk; $7=$ not at all at risk)

\section{RISK TO PEERS}

If some other person your age engaged in this activity, to what extent do you believe that he/she would be at risk of getting hurt or getting sick? ( $1=$ very much at risk; $7=$ not at all at risk)

\section{BENEFITS VS RISKS}

To what extent are the benefits or pleasures provided by this type of activity greater than the risks associated with it? ( $1=$ risks much greater than the benefits; $7=$ benefits much greater than the risks)

\section{SERIOUSNESS OF EFFECTS}

If an accident or something bad happened because of this activity, would you expect the harmful effects to be mild, or serious? $(1=$ very mild harm; $7=$ very serious harm $)$ 
Psychometric Study of Adolescent Risk Perception

Benthin, Slovic, Severson / page 25

Table 2, continued

\section{INFORMATION VALUE}

If an accident or something bad happened because of this activity, to what extent would it provide important information to young people that similar or even worse things might happen in the future? $(1=$ low information value; $7=$ high information value)

\section{PERSONAL CONTROL}

If someone your age did this activity, to what extent could he or she control the risks associated with it? ( 1 = risks cannot be controlled; 7 = risks can be completely controlled)

\section{PEER INFLUENCE}

To what extent are you influenced by your friends to do this activity? ( 1 = not at all influence; $7=$ greatly influenced)

\section{ADMIRATION}

To what extent are teenagers who are doing this activity admired by their peers? $(1=$ not at all admired; 7 = greatly admired)

\section{NEED FOR REGULATION}

To what extent does this type of activity need to be controlled by parents, school officials, or the law? $(1=$ little or no regulation needed; $7=$ very strict regulation needed)

\section{AVOIDABILITY}

To what extent can a person your age avoid doing this activity? $(1=$ cannot be avoided; $7=$ can be avoided)

PERCEIVED PARTICIPATION

What percentage of people your age would you say are doing this activity? (00 to 100\%) 
Table 3. Means, Standard Deviations, and Extreme Activities for Each Scale

\begin{tabular}{lccccl}
\hline \hline \multicolumn{1}{c}{ Scale } & Mean & SD & & \multicolumn{2}{c}{ Extreme Activities } \\
\hline Old/New Risk & 4.71 & 1.14 & $(1.72)$ Crack & $\rightarrow(6.68)$ Swimming \\
Knowledge & 4.01 & 0.81 & $(2.54)$ Diet Pills $\rightarrow(5.22)$ Sex \\
Fear & 3.35 & 1.53 & $(1.22)$ Bicycle Riding $\rightarrow(6.29)$ Crack \\
Personal Risk & 3.95 & 1.49 & $(1.37)$ Crack & $\rightarrow(6.51)$ Bicycle Riding \\
Risk to Peers & 3.94 & 1.51 & $(1.37)$ Crack & $\rightarrow(6.53)$ Bicycle Riding \\
Benefits vs. Risks & 3.77 & 1.73 & $(1.20)$ Cocaine & $\rightarrow(6.59)$ Bicycle Riding \\
Seriousness of Effects & 5.01 & 1.28 & $(2.78)$ Caffeine & $\rightarrow(6.98)$ Crack \\
Information Value & 3.99 & 0.99 & $(2.50)$ Contact Sports $\rightarrow(5.85)$ Uppers \\
Personal Control & 3.74 & 0.96 & $(1.95)$ Crack & $\rightarrow(5.10)$ Bicycle Riding \\
Peer Influence & 2.44 & 0.88 & $(1.34)$ Uppers & $\rightarrow(4.20)$ Driving \\
Admiration & 3.20 & 1.28 & $(1.42)$ Binge Eating $\rightarrow(5.80)$ Driving \\
Need for Regulation & 3.96 & 1.65 & $(1.51)$ Bicycle Riding $\rightarrow(6.71)$ Cocaine \\
Avoidability & 5.73 & 0.83 & $(4.24)$ Driving & $\rightarrow(6.78)$ Crack \\
Perceived Participation & 50.80 & 23.60 & $(18.50)$ Binge Eating $\rightarrow(85.90)$ Caffeine \\
& & & &
\end{tabular}

Note: In some cases, high ratings are associated with low perceptions of the quality (e.g., personal risk). The labeling of each scale is given in Table 2 . 
Table 4. Intercorrelations among 11 High-Risk Activities across 41 Subjects

\begin{tabular}{|c|c|c|c|c|c|c|c|c|c|c|c|}
\hline & 1 & 2 & 3 & 4 & 5 & 6 & 7 & 8 & 9 & 10 & 11 \\
\hline 1. Alcohol & -- & & & & & & & & & & \\
\hline 2. Five or More Drinks & .63 & -- & & & & & & & & & \\
\hline 3. Rode with Drunk & .44 & .38 & --- & & & & & & & & \\
\hline 4. Sex & .21 & .19 & .52 & --- & & & & & & & \\
\hline 5. Unprotected Sex & .03 & .17 & .13 & .62 & -- & & & & & & \\
\hline 6. Cigarettes & .32 & .59 & .21 & .27 & .31 & -- & & & & & \\
\hline 7. Marijuana & .57 & .51 & .56 & .36 & .21 & .45 & -- & & & & \\
\hline 8. Snuff & .08 & .23 & .23 & .70 & .88 & .39 & .27 & -- & & & \\
\hline 9. Uppers & .15 & .01 & .33 & .43 & .31 & .09 & .03 & .26 & $\cdots$ & & \\
\hline 10. Cocaine & .10 & .16 & .23 & .30 & .48 & .23 & .18 & .42 & .70 & -- & \\
\hline 11. Crack & .10 & -.15 & .23 & .30 & -.05 & -.11 & -.14 & -.06 & .70 & -.02 & -- \\
\hline
\end{tabular}

